\title{
COHOMOLOGICAL TRIVIALITY BY SPECTRAL METHODS
}

\author{
JUAN JOSÉ MARTÍNEZ ${ }^{1}$
}

\begin{abstract}
In this note, the spectral sequence of a group extension is used to obtain a direct proof of the twins' criterion for cohomological triviality of modules over a finite group, stated in its strong form.
\end{abstract}

The Tate cohomology of finite groups is denoted by $\hat{H}$, while $H$ is reserved for the ordinary group cohomology.

The criterion to be proved is the following.

TheOREM (NAKAYAMA-TATE). Let $G$ be a finite group and $A$ a $G$-module. If, for each prime $p$, there exists an integer $r_{p}$ (depending on $p$ ) such that

$$
\hat{H}^{r_{p}}\left(S_{p}, A\right)=\hat{H}^{r_{p}+1}\left(S_{p}, A\right)=0,
$$

where $S_{p}$ is a Sylow p-subgroup of $G$, then $A$ is cohomologically trivial.

Proof. As usual, by the Sylow subgroup argument in cohomology [3, Corollary to Theorem 4, p. 148], it suffices to consider the case where $G$ is a $p$-group, then showing that the $G$-module $A$ is cohomologically trivial if $\hat{H}^{r}(G, A)=\hat{H}^{r+1}(G, A)=0$ for some integer $r$. This is an immediate consequence of the following two statements:

(i) If $\hat{H}^{r}(G, A)=\hat{H}^{r+1}(G, A)=0$, then $\hat{H}^{r}(S, A)=\hat{H}^{r+1}(S, A)=0$ for all subgroups $S$ of $G$.

(ii) If $\hat{H}^{r}(G, A)=\hat{H}^{r+1}(G, A)=0$, then $\hat{H}^{n}(G, A)=0$ for all integers $n$.

Since every proper subgroup of $G$ is contained in a normal subgroup of index $p$ in $G$, to establish (i) $S$ can be taken as such a subgroup, arguing by induction on the order of $G$. Also, by the standard technique of dimension shifting [3, §1, p. 137], it can be assumed that $r=1$.

Now, let $(E, H)$ be the Hochschild-Serre spectral sequence associated with the $G$-module $A$ and the subgroup $S$ of $G$ [2], so that

$$
E_{2}^{p, q}=H^{p}\left(G / S, H^{q}(S, A)\right) \text { and } H^{n}=H^{n}(G, A) .
$$

Since $H^{1}=0, H^{1}(S, A)_{G / S} \simeq E_{2}^{1,1}, G / S$ being a cyclic group. For applying the formula $\operatorname{res}_{G, S} \operatorname{cor}_{S, G}=N_{G / S}[1$, Corollary 9.2, p. 257] for dimension 1, $N_{G / S} H^{1}(S, A)=0$, and so, $H^{1}(S, A)_{G / S} \simeq \hat{H}^{-1}\left(G / S, H^{1}(S, A)\right) ;$ but $\hat{H}^{-1}\left(G / S, H^{1}(S, A)\right) \simeq E_{2}^{1,1}$, by the periodicity of the cohomology of finite cyclic groups [3, Corollary to Proposition 6, p. 141]. From $H^{1}=0$ it also

Received by the editors January 3, 1978.

AMS (MOS) subject classifications (1970). Primary $18 \mathrm{H} 10$.

Key words and phrases. Criterion for cohomological triviality, spectral sequence of a group extension.

${ }^{1}$ Miembro de la Carrera del Investigador del CONICET. 
follows that $E_{2}^{1,1} \simeq E_{3}^{1,1}$, because $E_{2}^{1,0}=0$ and hence, $E_{2}^{3,0}=0$ (periodicity again). Moreover, $E_{3}^{1,1}=0$, since $H^{2}=0$. Therefore, it has been proved that $H^{1}(S, A)_{G / S}=0$. Now, since $H^{1}(S, A)$ is annihilated by a power of $p$ [3, Corollary 1 to Proposition 4, p. 138], a property of finite $p$-groups yields $H^{1}(S, A)=0$. (This property is well known, at least for modules annihilated by $p$ [3, Lemma 4, p. 149]; the case of a module $M$ annihilated by a $p$-power reduces to the elementary case, by considering $M / p M$.) Finally, since $H^{1}(S, A)=0$, the spectral sequence provides an exact sequence $H^{2} \rightarrow E_{2}^{0,2} \rightarrow$ $E_{2}^{3,0}$, where the extreme terms vanish. Thus, $E_{2}^{0,2}=0$, which implies $H^{2}(S, A)$ $=0$. (This follows from the fact that, if a $p$-primary module $M$ over a finite $p$-group $K$ satisfies $M^{K}=0$, then $M=0$. Since $M=U N$, where $N$ runs through all finitely generated subgroups of $M, M^{K}=\cup N^{K}$, and the result can be deduced from the finite case [3, Lemma 2, p. 146].)

By dimension shifting and by moving up or down one dimension at a time, to prove (ii) it is sufficient to show that:

(iii) If $H^{1}(G, A)=H^{2}(G, A)=0$, then $H^{3}(G, A)=0$.

(iv) If $H^{2}(G, A)=H^{3}(G, A)=0$, then $H^{1}(G, A)=0$.

Proceeding by induction on the order of $G$, given a normal subgroup $S$ of index $p$ in $G$, in the case of (iii) it follows that $H^{3}(S, A)=0$, because $H^{1}(S, A)=H^{2}(S, A)=0$, by (i). Then, $E_{2}^{3,0} \simeq H^{3}$ (at this point, the spectral sequence is not essential [3, Corollary to Proposition 5 (Proof), p. 126]); but $E_{2}^{3,0}=0$, as before, since $H^{1}=0$. Similarly, under the hypothesis of (iv), assertion (i) gives $H^{2}(S, A)=H^{3}(S, A)=0$, and hence, by the inductive assumption, $H^{1}(S, A)=0$. Therefore, $E_{2}^{3,0} \simeq H^{3}=0$, so that $H^{1} \simeq E_{2}^{1,0}=$ 0 . Thus, the proof of the theorem is complete.

\section{REFERENCES}

1. H. Cartan and S. Eilenberg, Homological algebra, Princeton Univ. Press, Princeton, N. J., 1956. MR 17, 1040.

2. G. Hochschild and J.-P. Serre, Cohomology of group extensions, Trans. Amer. Math. Soc. 74 (1953), 110-134. MR 14, 619.

3. J.-P. Serre, Corps locaux, Hermann, Paris, 1962. MR 27 \#133.

Departamento de Matemáticas, Facultad de Ciencias Exactas y Naturales, UniversiDad de Buenos Aires, 1428 Buenos Aires, Argentina 